\title{
Enhancer of zeste homolog 2 depletion arrests the proliferation of hepatoblastoma cells
}

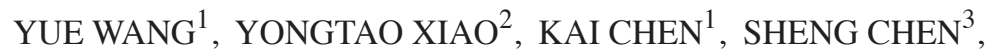 \\ MIN ZHANG ${ }^{1}$, ZHIXIANG WU ${ }^{1}$ and YEMING WU ${ }^{1}$ \\ ${ }^{1}$ Department of Pediatric Surgery, Xin Hua Hospital Affiliated to Shanghai Jiao Tong University School of Medicine, \\ Shanghai 200092; ${ }^{2}$ Shanghai Institute for Pediatric Research, Shanghai $200092 ;{ }^{3}$ Department of Pediatric Surgery, \\ Shanghai Children's Medical Center, Affiliated to Shanghai Jiao Tong University School of Medicine,
}

Shanghai 200127, P.R. China

Received April 7, 2015; Accepted January 8, 2016

DOI: $10.3892 / \mathrm{mmr} .2016 .4864$

\begin{abstract}
Hepatoblastoma is the most common type of malignant liver tumor in children. While outcomes have been greatly improved in the past decades, the treatment of advanced hepatoblastoma has remained challenging. Enhancer of zeste homologue 2 (EZH2), a member of the polycomb group regulators of gene activity, is amplified and overexpressed in a variety of cancers. However, the role of EZH2 in hepatoblastoma has remained to be fully elucidated. The purpose of the present study was to investigate the expression patterns of EZH2 in hepatoblastoma cells and to assess the anti-cancer effects of EZH2 depletion. Western blot analysis revealed that EZH2 expression was significantly higher in hepatoblastoma specimens compared with that in peri-tumor tissues, while p27 was reduced in hepatoblastoma. Suppression of EHZ2 using lentiviral small hairpin RNA inhibited hepatoblastoma cell proliferation, induced cell cycle arrest in G1 phase and enhanced the expression of G1/S-phase checkpoint protein p27. These results suggested that EZH2 may represent a potential diagnostic marker and therapeutic target for the treatment of hepatoblastoma.
\end{abstract}

\section{Introduction}

Hepatoblastoma is the most common type of malignant liver tumor in children, accounting for $\sim 62 \%$ of primary hepatic malignant tumors. The etiology and pathogenetic mechanisms of hepatoblastoma have remained to be fully elucidated; however, it has been indicated that chromosomal abnormalities,

Correspondence to: Professor Yeming Wu, Department of Pediatric Surgery, Xin Hua Hospital Affiliated to Shanghai Jiao Tong University School of Medicine, 1665 Kongjiang Road, Yangpu, Shanghai 200092, P.R. China

E-mail:wuymsh@163.com

Key words: enhancer of zeste homolog 2, depletion, hepatoblastoma, lentiviral small hairpin RNA, p27 genetic factors, low birth weight and the adverse external factors during pregnancy may be factors associated with the occurrence of hepatoblastoma (1-3). Surgery is currently the most effective method for treating hepatoblastoma. However, as it is difficult to detect hepatoblastoma in early stages, approximately half of the patients with hepatoblastoma present with an unresectable tumor at the time-point of diagnosis (4). Therefore, it is urgently required to develop potential diagnostic biomarkers and therapeutic targets for hepatoblastoma.

Enhancer of zeste homolog 2 (EZH2) is a sub-unit of the polycomb-repressive complex 2, which also contains EED and SUZ12, catalyzes the trimethylation of histone $\mathrm{H} 3$ on Lys 27 and mediates transcriptional silencing (5). EZH2 is overexpressed in a variety of cancer types, including prostate, breast, gastric and bladder carcinomas (6). Overexpression of EZH2 has been associated with the invasion and progression of malignant cancers, particularly with the progression of prostate cancer (7). EZH2 has also been reported to be overexpressed in hepatocellular carcinomas, in which it has an oncogenic function (8). Of note, EZH2 was found to be a promising biomarker and associated with the progression and aggressive biological behavior of hepatocellular carcinoma (9). Hajósi-Kalcakosz et al (10) previously demonstrated that EZH2 is positively expressed in hepatoblastoma using immunohistochemistry. However, the roles of EZH2 protein in hepatoblastoma, the most common malignant liver cancer in children, have remained largely elusive.

The present study therefore investigated the expression and the roles of EZH2 protein in the proliferation of hepatoblastoma. It was revealed that $\mathrm{EZH} 2$ was overexpressed in hepatoblastoma tissues; furthermore, loss-of-function studies using small hairpin (sh)RNA-mediated knockdown of EZH2 indicated a potential oncogenic function of this gene by driving the proliferation of hepatoblastoma cell lines.

\section{Materials and methods}

Tissue samples. Seven surgical specimens of hepatoblastoma were collected and analyzed in the present study. The peri-tumor tissues were used as controls. Patient details are listed in Table I. Written informed consent was obtained from 
Table I. Characteristics of patients diagnosed with hepatoblastoma.

\begin{tabular}{lcclccc}
\hline $\begin{array}{l}\text { Patient } \\
\text { No. }\end{array}$ & $\begin{array}{c}\text { Gender } \\
(\mathrm{M} / \mathrm{F})\end{array}$ & $\begin{array}{c}\text { Age } \\
(\mathrm{M})\end{array}$ & $\begin{array}{c}\text { Presenting } \\
\text { symptom }\end{array}$ & $\begin{array}{c}\text { Clinical } \\
\text { stage }\end{array}$ & $\begin{array}{c}\text { Metastasis } \\
(\mathrm{Y} / \mathrm{N})\end{array}$ & $\begin{array}{c}\text { Preoperative } \\
\text { chemotherapy }(\mathrm{Y} / \mathrm{N})\end{array}$ \\
\hline 1 & $\mathrm{M}$ & 8 & Abdominal mass & III & $\mathrm{N}$ & $\mathrm{Y}$ \\
2 & $\mathrm{M}$ & 96 & Abdominal pain & IV & $\mathrm{Y}$ & $\mathrm{N}$ \\
3 & $\mathrm{M}$ & 11 & Jaundice & III & $\mathrm{N}$ & $\mathrm{N}$ \\
4 & $\mathrm{M}$ & 5 & Abdominal mass & III & $\mathrm{N}$ & $\mathrm{Y}$ \\
5 & $\mathrm{M}$ & 14 & Abdominal mass & III & $\mathrm{N}$ & $\mathrm{Y}$ \\
6 & $\mathrm{~F}$ & 18 & Abdominal mass & III & $\mathrm{N}$ & $\mathrm{Y}$ \\
7 & $\mathrm{~F}$ & 21 & Abdominal mass & IV & $\mathrm{Y}$ & $\mathrm{Y}$ \\
\hline
\end{tabular}

Y, Yes; N, no; M, months.

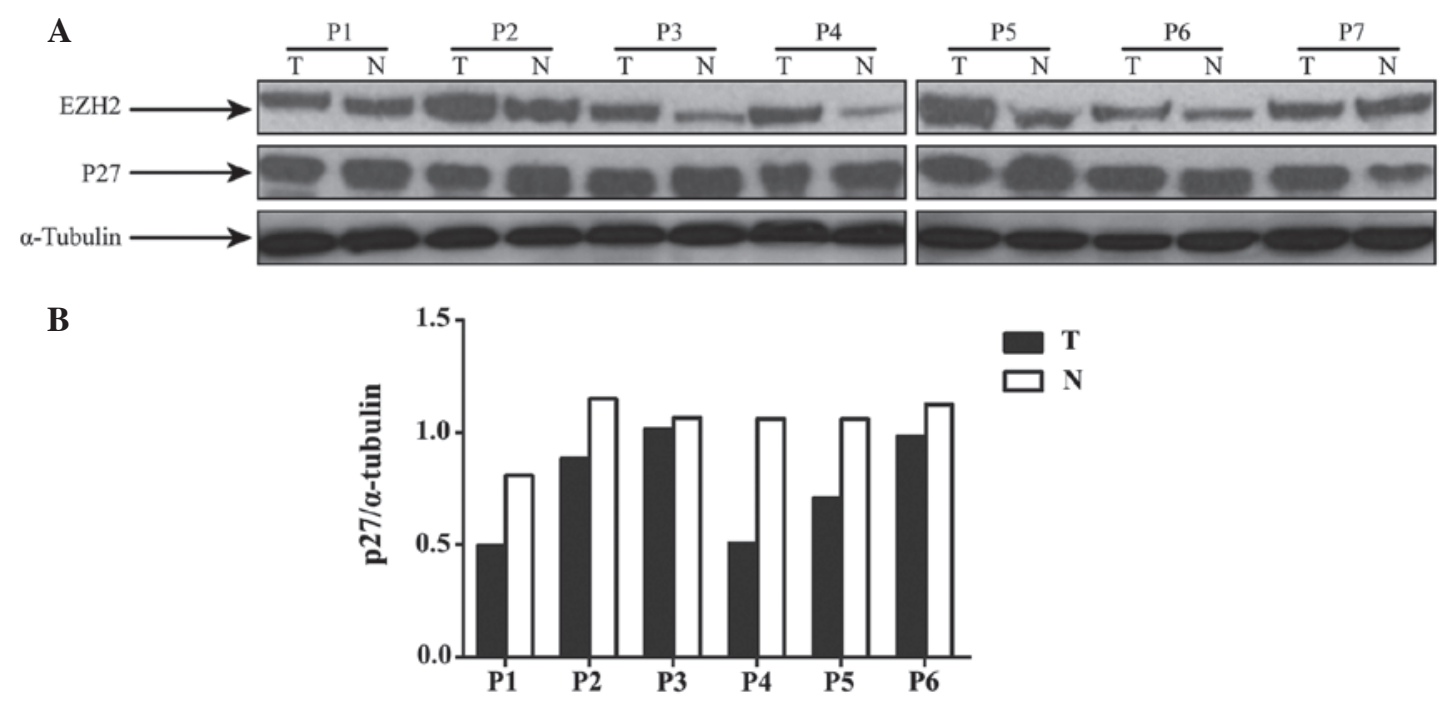

Figure 1. (A) EZH2 expression in hepatoblastoma specimens is higher than that in normal tumor-surrounding liver tissues in patients 1-7 according to western blot analysis with $\alpha$-Tubulin as a loading control. p27 expression in hepatoblastoma was downregulated in 6 out of 7 patients compared with normal peri-tumor tissues. (B) Quantified results of p27/ $\alpha$-tubulin in patients 1-6. T, tumor tissue, N, normal tissue, P, patient; EZH2, enhancer of zeste homolog 2.

the patients' guardians. The present study was approved by the ethics committee of Xinhua Hospital, Affiliated to the School of Medicine, Shanghai Jiaotong University (Shanghai, China).

Cell culture. The human embryonic kidney (HEK)293T cell line and the Huh6 and HepG2 hepatoblastoma cell lines were obtained from the Cell Bank of the Chinese Academy of Sciences (Shanghai, China) and cultured in Dulbecco's modified Eagle's medium (Gibco; Thermo Fisher Scientific, Inc., Waltham, MA, USA) supplemented with $10 \%$ fetal bovine serum (Gibco; Thermo Fisher Scientific, Inc.). All cultures were maintained in a humidified atmosphere containing $5 \%$ $\mathrm{CO}_{2}$ at $37^{\circ} \mathrm{C}$.

shRNA, lentiviral construction and transduction. The shRNA targeting of human EZH2 was performed as described previously (8). Briefly, the synthesized sequence, 5'-GAC TCTGAATGCAGTTGCTTCAGTACCC-3', against EZH2 (GeneChem Co., Ltd., Shanghai, China) were inserted into the pGCSIL-enhanced green fluorescent protein (EGFP) vector (GeneChem Co., Ltd.) via its AgeI and EcoRI sites
(New England BioLabs, Inc., Ipswich, MA, USA). A negative sequence used as a control was inserted into the identical plasmid at the identical site. The resulting lentiviral vector expressing the EZH2 siRNA or negative control siRNA was co-transfected into HEK293T cells with Lipofectamine 2000 (Invitrogen, Thermo Fisher Scientific, Inc.) according to the manufacturer's instructions. The viral titer was determined by counting EGFP-positive cells under a MicroPublisher 3.3 RTV fluorescence microscope (Olympus Corporation, Tokyo, Japan). The supernatant of the transfected HEK293T cells, which contained the lentiviral vector, was used to transduce $1 \times 10^{5} / \mathrm{cm}^{2}$ Huh6 and HepG2 cells in the presence of polybrene (10 $\mu \mathrm{g} / \mathrm{ml}$; GeneChem Co., Ltd.). Cells and supernatants were harvested at different time-points (24, 48, 72 and $96 \mathrm{~h})$ after transduction.

Cell proliferation assay. The stably transfected cells were plated into 96 -well plates at a density of $1 \times 10^{4}$ per well. The medium in each well was replaced daily. Numbers of cells were detected at 0,48 or $96 \mathrm{~h}$ post-transfection using a Cell Counting Kit-8 (CCK-8; Dojindo Molecular Technologies, 

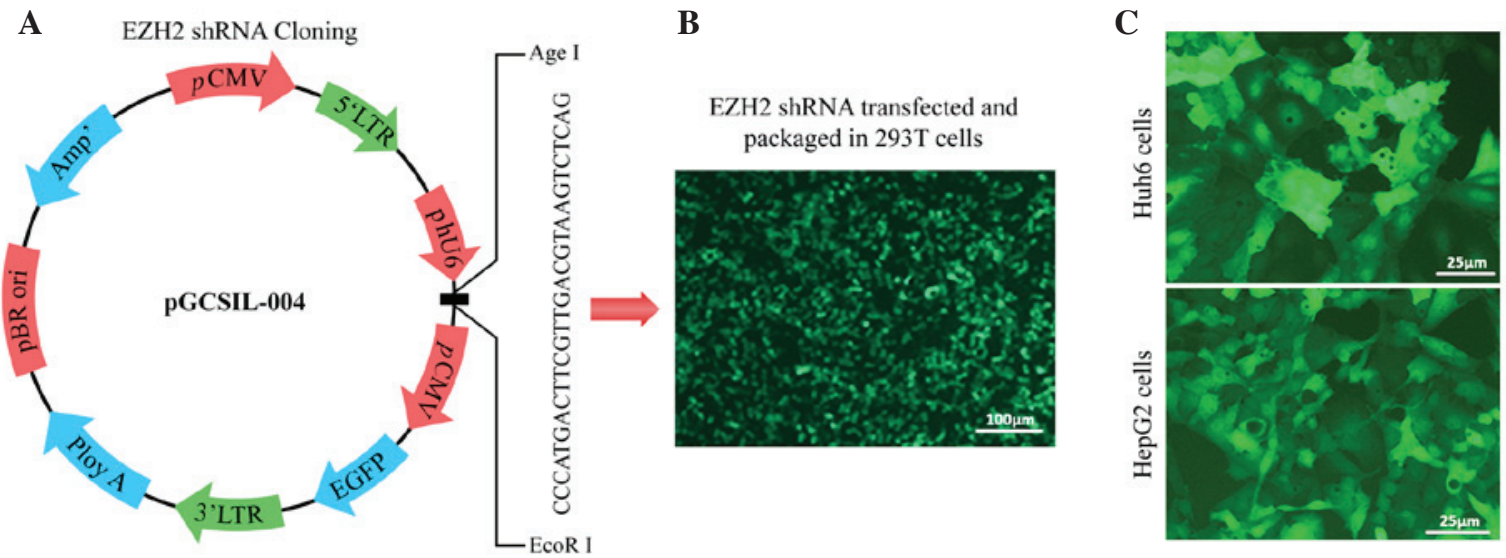

Figure 2. Efficient suppression of EZH2 by lenti-shRNA in hepatoblastoma cells. (A) shRNAs targeting human EZH2 were inserted into the pGCSIL-EGFP vector via its $A g e I$ and EcoRI sites. (B) Efficient transduction of HEK293T cells with lenti-shRNA (magnification, x100) and (C) efficient infection of Huh6 and HepG2 cells with lenti-shRNA (magnification, x400) were confirmed by fluorescence microscopy. LTR, long terminal repeat; pCMV, cytomegaloviral plasmid; EGFP, enhanced green fluorescence protein; lenti-shRNA, lentiviral small hairpin RNA; EZH2, enhancer of zeste homolog 2.

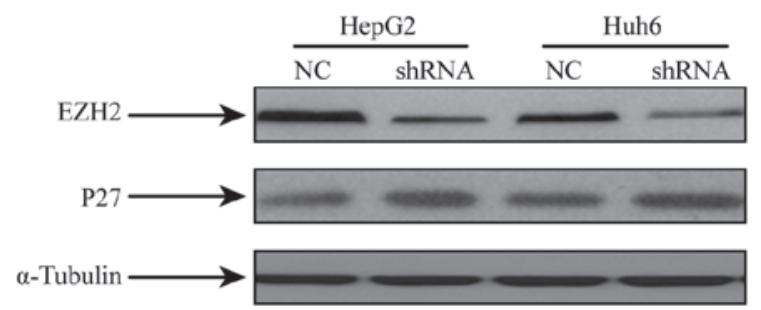

Figure 3. EZH2 protein levels were decreased in Huh6 and HepG2 cells transfected with lentiviral shRNA compared to those in NC cells as indicated by western blot analysis with $\alpha$-Tubulin as a loading control. P27 expression was increased in cells with EZH2 repression compared to that in NC cells. $\mathrm{NC}$, negative control; shRNA, small hairpin RNA; EZH2, enhancer of zeste homolog 2 .
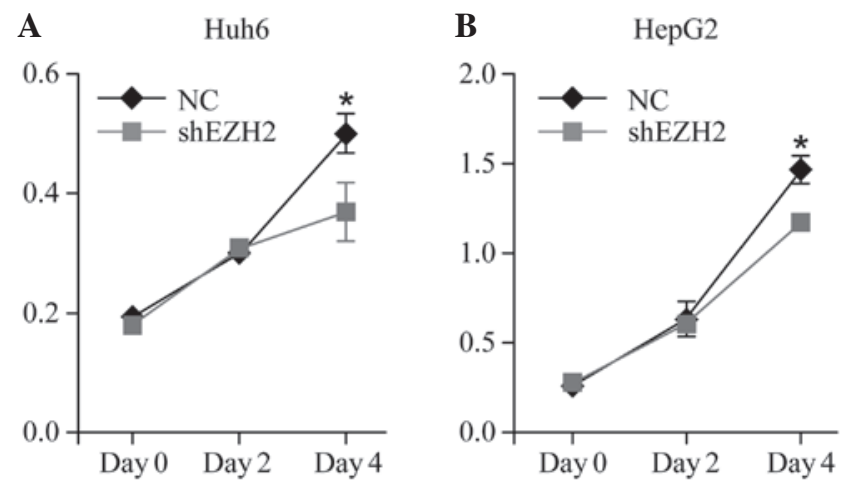

Figure 4. Suppression of EZH2 by lentiviral shRNA inhibits hepatoblastoma cells growth in vitro. Knockdown of EZH2 inhibited the proliferation of (A) Huh6 cells (NC, $0.36 \pm 0.05$ vs. shEZH2, 0.50 \pm 0.01 ) (B) and HepG2 cells (NC, $1.17 \pm 0.02$ vs. shEZH2, $1.49 \pm 0.01$ ). Results are expressed as the mean \pm standard deviation. ${ }^{*} \mathrm{P}<0.05$ vs. shEZH 2 group. shEZH 2 , small hairpin RNA targeting enhancer of zeste homolog 2; NC, negative control.

Kumamoto, Japan) according to the manufacturer's instructions. At $2 \mathrm{~h}$ following addition of the CCK- 8 stain, the absorbance values of the wells were measured at $450 \mathrm{~nm}$ using a microplate reader (Synergy ${ }^{\mathrm{TM}}$ H1 Multi-Mode Reader; BioTek Instruments, Inc., Winooski, VT, USA). Each experiment was performed in triplicate.
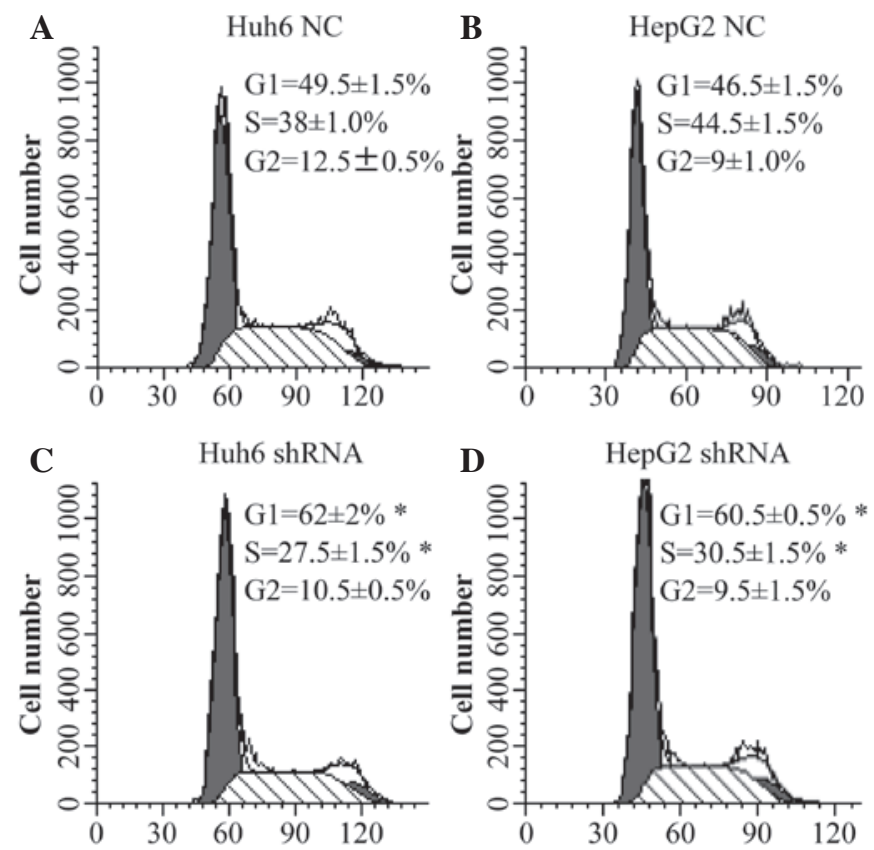

Figure 5. EZH2 repression significantly increases the G1 population, accompanied by a decrease of the $\mathrm{S}$-phase population, in hepatoblastoma cell lines. (A and B) Cell cycle distribution in NC groups of Huh6 and HepG2 cells. (C and D) EZH2 depletion induced cell cycle arrest in G1 phase in Huh6 and HepG2 cells. * $\mathrm{P}<0.05$ vs. the NC group. NC, negative control; shRNA, small hairpin RNA; EZH2, enhancer of zeste homolog 2.

Protein extraction and western blot analysis. Western blotting was used to determine EZH2 and p27 expression levels in extracts from Huh6 and HepG2 cells grown in a six-well plate. The cells were washed twice with cold phosphate-buffered saline (PBS) prior to addition of radioimmunoprecipitation assay lysis buffer containing protease inhibitors (Thermo Fisher Scientific, Inc.). Extracts were centrifuged at $12,000 \mathrm{x} \mathrm{g}$ for $15 \mathrm{~min}$ at $4^{\circ} \mathrm{C}$ and the supernatant was collected. The protein concentration was determined using a bicinchoninic acid protein assay (Thermo Fisher Scientific, Inc.). Forty micrograms of extracted protein were purified by $12 \%$ sodium dodecyl sulfate-polyacrylamide 
gel electrophoresis (Invitrogen; Thermo Fisher Scientific, Inc.) and electrophoretically transferred onto nitrocellulose membranes. The membranes were blocked overnight in $5 \%$ bovine serum albumin for $2 \mathrm{~h}$, washed 3 times for 5 min in PBS containing 0.1\% Tween 20 (Shanghai Yeasen Biotechnology Co., Ltd., Shanghai, China). The membranes were then incubated with primary antibodies at $4^{\circ} \mathrm{C}$ overnight. The primary antibodies used were all obtained from Cell Signaling Technology, Inc. (Danvers, MA, USA) and were as follows: Rabbit monoclonal anti-EZH2 (1:1,000; cat. no. 5246), rabbit monoclonal anti-p27 (1:1,000; cat. no. 3686) and rabbit polyclonal anti- $\alpha$-tubulin (1:1,000; cat. no. 2148) antibodies. Subsequently, membranes were incubated with a goat anti-rabbit IgG coupled with horseradish peroxidase (1:2,000; Cell Signaling Technology, Inc.; cat. no. 7074) for $2 \mathrm{~h}$ at room temperature. Immunoreactivity was visualized using enhanced chemiluminescence reagents (SuperSignal West Pico; Thermo Fisher Scientific, Inc.) and Kodak X-OMAT film (Kodak, Rochester, NY, USA) with a ChemiDoc XRS molecular imager (Bio-Rad Laboratories, Inc., Hercules, CA, USA) used to capture the images.

Flow cytometric analysis of cell cycle distribution. Equal numbers of Huh6 and HepG2 cells were seeded in a 12-well plate. Seventy-two hours after transfection, cells were trypsinized and fixed with $70 \%$ ethanol overnight and stained with $100 \mu \mathrm{g} / \mathrm{ml}$ propidium iodide (PI; R\&S Biotech, Shanghai, China) containing $100 \mu \mathrm{g} / \mathrm{ml}$ RNase (DNase free; Shanghai Yeasen Biotechnology Co., Ltd.). After incubation at $37^{\circ} \mathrm{C}$ for $30 \mathrm{~min}$, the samples were analyzed on BD FACSCanto II flow cytometer (BD Biosciences, Franklin Lakes, NJ, USA). The average percentages of cells in G1, S or G2 phases of the cell cycle were quantified and the standard error was calculated for three experiments.

Statistical analysis. All data were reported as the mean \pm standard error of the mean. Statistical analysis was performed using SPSS version 19.0 (International Business Machines, Armonk, NY, USA). Student's t-tests (two-tailed, unpaired) was performed for comparison between groups. $\mathrm{P}<0.05$ was considered to indicate a statistically significant difference.

\section{Results}

EZH2 is overexpressed in hepatoblastoma tissues. Seven patients with hepatoblastoma were enrolled in the present study (Table I). Their age ranged from five months to eight years and five of the patients were male. Their presenting symptoms included a palpable abdominal mass, abdominal pain and jaundice. Five of the patients had received four courses of pre-operative chemotherapy to enable their tumors to be resected. The remaining patients received primary surgery with post-operative chemotherapy.

The hepatoblastoma tissues and adjacent normal tissues were assessed for the expression of EZH2 using western blot analysis. As shown in Fig. 1, EZH2 was found to be overexpressed in hepatoblastoma tissues compared with that in normal peri-tumor tissues. This upregulation of EZH2 in hepatoblastoma tissues indicated its potential oncogenic roles in hepatoblastoma. Therefore, the function of $\mathrm{EZH} 2$ was further assessed in hepatoblastoma cell lines in vitro by using shRNA-mediated depletion of EZH2.

shRNA-mediated suppression of EZH2 in hepatoblastoma cell lines. To further study the biological roles of EZH2 in hepatoblastoma, a lentiviral shRNA system was applied (Fig. 2A and B). Huh6 and HepG2 cells were transduced with lentivirus expressing shRNA targeting EZH2 or empty vector (Fig. 2C). A marked knockdown of EZH2 protein expression was detected at $48 \mathrm{~h}$ after transfection (Fig. 3).

EZH2 deletion inhibits hepatoblastoma cell proliferation. To assess the potential effects of shRNA-mediated EZH2 silencing on the proliferation of hepatoblastoma cells, a CCK-8 assay was performed. As shown in Fig. 4, transfection with shRNA targeting EZH2 caused a significant reduction in the proliferation of the Huh6 and HepG2 cell lines compared with that in the $\mathrm{NC}$ groups $(\mathrm{P}<0.05)$. Huh6, NC, $0.36 \pm 0.05$ vs. shEZH2, $0.50 \pm 0.01$ and $\mathrm{HepG} 2, \mathrm{NC}, 1.17 \pm 0.02$ vs. $1.49 \pm 0.01$. These results suggested that $\mathrm{EZH} 2$ participates in the regulation of signaling cascades that positively influence hepatoblastoma cancer cell proliferation.

Inhibition of EZH2 results in $\mathrm{Gl}$ phase arrest in hepatoblastoma cells. To further explore the effects of EZH2 repression on the cell cycle, flow cytometric cell cycle analysis following PI staining was performed. The results clearly showed that EZH2 repression significantly increased the G1 populations, accompanied by a decrease in the S-phase populations, in Huh6 and HepG2 cells (Fig. 5). These results indicated that EZH2 depletion inhibited the proliferation of hepatoblastoma cells by blocking G1-to-S-phase transition.

Inhibition of EZH2 increases p27. Western blot analysis of p27 in hepatoblastoma specimens revealed a reduced expression compared to that in normal tissues (Fig. 1). To assess the effects of EZH2 on p27 expression, hepatoblastoma cells subjected to shRNA-mediated EZH2 silencing were analyzed for p27 expression by western blot analysis. It was revealed that the protein expression of p27 was increased in cells with $\mathrm{EZH} 2$ repression compared to that in the NC group (Fig. 3).

\section{Discussion}

The present study initially assessed the protein levels of EZH2 in hepatoblastoma tissues compared with those in adjacent normal liver tissues, revealing that EZH2 was significantly overexpressed in hepatoblastoma tissues. This result indicated that EZH2 is a potential oncogene and tumor-specific marker in hepatoblastoma. To further study the role of EZH2 in hepatoblastoma, EZH2 was silenced in the Huh6 and HepG2 hepatoblastoma cell lines by using an effective lentiviral shRNA system. A significant inhibition of cell growth was observed in vitro following knockdown of EZH2 expression, which was consistent with previous observations in hepatocellular carcinoma $(8,11)$.

Next, the present study examined the role of EZH2 in hepatoblastoma cells by observing the effects of EZH2 knockdown on the cell cycle. Silencing of EZH2 was found to inhibit G1-to-S-phase transition in vitro. Previous studies 
suggested that EZH2 is crucial for regulating the cell cycle by repressing several tumor suppressor genes, including p16, p27 and RUNX3 (12-15). As p27 is known to be a regulator of the G1/S-phase checkpoint (15), the present study investigated the protein levels of p27 in hepatoblastoma tissues as well as in cell lines following EZH2 knockdown. Compared to the normal tissues, p27 was shown to be downregulated in hepatoblastoma tissues, along with overexpression of EZH2. Of note, p27 was significantly upregulated following EZH2 knockdown in the Huh6 and HepG2 hepatoblastoma cell lines.

P27, a cyclin-dependent kinase inhibitor, has been reported as a target gene of EZH2 in several carcinoma types $(15,16)$. In line with these findings, the present study confirmed that in hepatoblastoma tissues with EZH2 overexpression, p27 expression was downregulated, and that depletion of EZH2 in hepatoblastoma cell lines increased the expression of p27. These results suggested that EZH2 contributed to hepatoblastoma cell proliferation in part through epigenetic silencing of p27.

In conclusion, the present study demonstrated that EZH2 is upregulated in hepatoblastoma. It exerts its oncogenic function at least in part by inhibiting p27 expression and thereby reducing the proliferation of hepatoblastoma cells by blocking G1-to-S-phase transition. These results indicated that EXH2 may represent a diagnostic biomarker and a potential therapeutic target for the treatment of hepatoblastoma.

\section{References}

1. Yang SS, Brough AJ and Bernstein J: Tumors of the liver in early infancy: Hepatoblastoma. Mich Med 71: 539-543, 1972.

2. Rowland JM: Hepatoblastoma: Assessment of criteria for histologic classification. Med Pediatr Oncol 39: 478-483, 2002.

3. Malogolowkin MH, Katzenstein HM, Krailo M and Meyers RL: Treatment of hepatoblastoma: The North American cooperative group experience. Front Biosci (Elite Ed) 4: 1717-1723, 2012.

4. Warmann SW and Fuchs J: Drug resistance in hepatoblastoma. Curr Pharm Biotechnol 8: 93-97, 2007.
5. Cao R, Wang L, Wang H, Xia L, Erdjument-Bromage H, Tempst $P$, Jones RS and Zhang Y: Role of histone H3 lysine 27 methylation in Polycomb-group silencing. Science 298: 1039-1043, 2002.

6. Xiao Y: Enhancer of zeste homolog 2: A potential target for tumor therapy. Int J Biochem Cell Biol 43: 474-477, 2011.

7. Varambally S, Dhanasekaran SM, Zhou M, Barrette TR, Kumar-Sinha C, Sanda MG, Ghosh D, Pienta KJ, Sewalt RG, Otte AP, et al: The polycomb group protein EZH2 is involved in progression of prostate cancer. Nature 419: 624-629, 2002.

8. Chen Y, Lin MC, Yao H, Wang H, Zhang AQ, Yu J, Hui CK, Lau GK, He ML, Sung J and Kung HF: Lentivirus-mediated RNA interference targeting enhancer of zeste homolog 2 inhibits hepatocellular carcinoma growth through down-regulation of stathmin. Hepatology 46: 200-208, 2007.

9. Cai MY, Tong ZT, Zheng F, Liao YJ, Wang Y, Rao HL, Chen YC, Wu QL, Liu YH, Guan XY, et al: EZH2 protein: A promising immunomarker for the detection of hepatocellular carcinomas in liver needle biopsies. Gut 60: 967-976, 2011.

10. Hajósi-Kalcakosz S, Dezső K, Bugyik E, Bödör C, Paku S, Pávai Z, Halász J, Schlachter K, Schaff Z and Nagy P: Enhancer of zeste homologue 2 (EZH2) is a reliable immunohistochemical marker to differentiate malignant and benign hepatic tumors. Diagn Pathol 7: 86, 2012.

11. Yonemitsu Y, Imazeki F, Chiba T, Fukai K, Nagai Y, Miyagi S, Arai M, Aoki R, Miyazaki M, Nakatani Y, et al: Distinct expression of polycomb group proteins EZH2 and BMI1 in hepatocellular carcinoma. Hum Pathol 40: 1304-1311, 2009.

12. Ougolkov AV, Bilim VN and Billadeau DD: Regulation of pancreatic tumor cell proliferation and chemoresistance by the histone methyltransferase enhancer of zeste homologue 2. Clin Cancer Res 14: 6790-6796, 2008.

13. Kotake Y, Cao R, Viatour P, Sage J, Zhang Y and Xiong Y: pRB family proteins are required for $\mathrm{H} 3 \mathrm{~K} 27$ trimethylation and Polycomb repression complexes binding to and silencing p16INK4alpha tumor suppressor gene. Genes Dev 21: 49-54, 2007.

14. Fujii S, Ito K, Ito Y and Ochiai A: Enhancer of zeste homologue 2 (EZH2) down-regulates RUNX3 by increasing histone H3 methylation. J Biol Chem 283: 17324-17332, 2008.

15. Nakagawa S, Okabe H, Sakamoto Y, Hayashi H, Hashimoto D, Yokoyama N, Sakamoto K, Kuroki H, Mima K, Nitta H, et al: Enhancer of zeste homolog 2 (EZH2) promotes progression of cholangiocarcinoma cells by regulating cell cycle and apoptosis. Ann Surg Oncol 20 (Suppl 3): S667-S675, 2013.

16. Sakurai T, Bilim VN, Ugolkov AV, Yuuki K, Tsukigi M, Motoyama T and Tomita Y: The enhancer of zeste homolog 2 (EZH2), a potential therapeutic target, is regulated by miR-101 in renal cancer cells. Biochem Biophys Res Commun 422: 607-614, 2012. 